\title{
Design of Hybrid NC Control System for Automatic Line
}

\author{
Feng Qian ${ }^{1, \mathrm{a}}, \mathrm{Ge} \mathrm{Xu}{ }^{1, \mathrm{~b}}$, Li Zhang ${ }^{1, \mathrm{c}}$ and Huimin Dong ${ }^{1, \mathrm{~d}}$ \\ 'Dalian University of Technology, Dalian 116024, China \\ ${ }^{2}$ FAW Jiefang Automotive Co. Ltd. of Wuxi, Wuxi 214026, China \\ qianfeng@dlut.edu.cn
}

\begin{abstract}
This paper presents a Hybrid NC Control System for Automatic line based on Cyber-Physical-System (CPS) technology. Firstly, this paper describes the overall system hierarchical structure, which uses a variety of techniques, such as sensors, smart computing, and heterogeneous network integration. In order to improve the practical value, we study and solve some technical problems, such as heterogeneous systems integration, data storage, and real-time simulation. Finally, the prototype system is developed, which has been applied in a large State-owned enterprise in Wuxi, China.
\end{abstract}

Keywords: Manufacturing systems; cyber-physical systems; distributed systems

\section{Introduction}

NC production line is an important symbol of industrial modernization, is an important means to improve the market competitiveness of enterprises. In discrete manufacturing enterprises, most of $\mathrm{NC}$ production lines exist as relatively independent manufacturing cells or manufacturing areas, typically containing processing equipment, Logistics equipment, warehousing equipment, inspection equipment and other auxiliary equipment. How to fully tap the potential of CNC production lines to reduce costs and improve competitiveness of enterprises is an important task.

The current research for NC line focuses on modeling and simulation, process-monitoring area. Some scholars study the production simulation based on virtual reality technology, analysis of the bottlenecks, receiving good results [1-6]. Some scholars experience in study process monitoring based on PLC technology, production line equipment with a sensor network connection, providing a basis for automatic remote control [7-11].

Amount due to the long preparation period, programming, human-computer, these studies generally used for mass production of process manufacturers. Discrete manufacturing production is characterized by multiple varieties, small batch. And it is difficult to apply the aforementioned research results. Discrete manufacturing CNC production lines have strong Hybrid Characteristic. The main causes of the problem are the NC production line with hybrid properties of the following features:

- It has a hybrid hardware structure. It is generally in accordance with the procedure characteristics formed by a mixture of different vendors' devices. According to the needs of the market, may increase, reduce some, resulting in unstable internal of the production line.

- It uses various hybrid operating systems. It contains universal CNC system (such as Fanuc, Siemens, and so on), General PLC system (such as Omron) and Dedicated PLC systems.It has a hybrid data boundary. According to statistics, about more than 40 kinds of fieldbus technology in the world [12-14], resulting in a heterogeneous network integration problem is particularly acute. 
- It has characteristics of hybrid production. Discrete manufacturing enterprise production requirements determined by the market, with larger fluctuations. It needs real time instead of a static model to guide production.

Therefore, research on the CNC production line for Discrete manufacturing enterprise problem is more complex, achieving similar process automation and unmanned autonomous control more difficult.

We study and design of a new Hybrid NC Control System (HNCS) based on Cyber Physical Systems (CPS) technology [15-19]. It will be armed for Intelligent Robot System for $\mathrm{CNC}$ production. And a new idea has been proposed on intelligent manufacturing research.

\section{System Design Requirement}

Units, intelligence, integration is the future development trend of manufacturing systems. Our system is to satisfy the requirements of a process of manufacturing units, and it has Intelligence, smart capacity to implement and intelligent decision. It can complete a specific task of an independent. According to production plan, it receives raw materials, transportation and processing until it is finished, and reporting the real state to the relevant system. When the system is designed, we focus on the following aspects:

- It should have good integration capabilities. It can achieve the integration of heterogeneous information of all kinds of NC equipment acquisition signal.

- In order to response NC equipment at millisecond speed, it needs to provide distributed embedded real-time control.

- It needs to provide a unified, standard operating environment for compatibility with a variety of commercial CNC system, sensors, and semantics.

- It can be used as building blocks of another system, and it has a metadata system describes the structure itself.

- It offers data exchange format based on XML, and Excel formats are supported too.

- It should provide fault tolerance verification mechanisms, data classification, data backup and recovery mechanisms authorized access mechanisms such as data-security measures.

- It should provide for remote network management, configuration management, and automated escalation capabilities.

\section{The Architecture of the System}

We design the system architecture is shown in Figure 1.

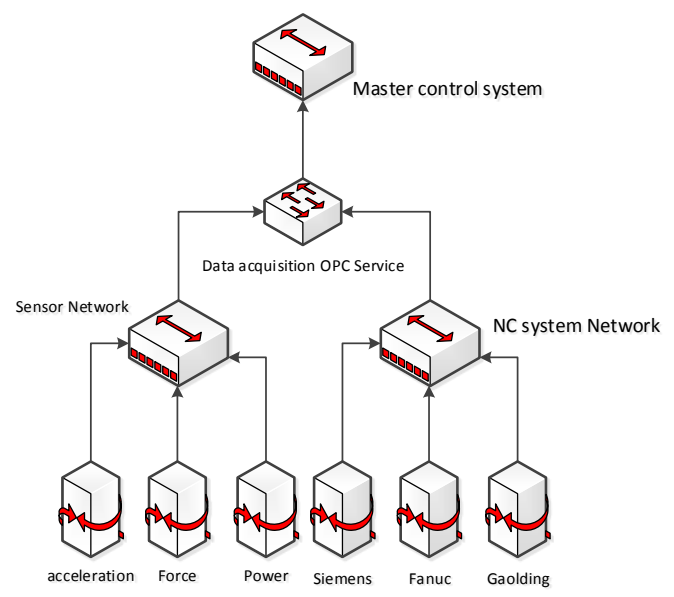

Figure 1. The Architecture of the System 
HNCS system using hierarchical design mainly includes the following levels:

- Perception and implementation layer. It is responsible for numerical control devices, sensors, signal acquisition, storage and transmission, and based on a strategy of real-time control of CNC equipment

- Gathering and storage layer. It is responsible for the heterogeneous networks, and unified the semantic model in the logical layer specification.

- Management and control layer. It is responsible for diagnosis and system improvement, system configuration, online tracking, remote, production system management functions, and provides the data input/output interface.

\subsection{Perception and Implementation Layer}

The layer is mainly responsible for acquisition, storage and transmission of sensor signals, and partial real-time control. Main module includes sensor configuration management, real time control system modules, data acquisition module.

3.1.1. Sensor Configuration Management Module: From the perspective of actual cost and integration, the system will prefer using CNC equipment built-in sensors. For NC devices do not provide sensors, our own purchase. Currently uses the following types of sensors:

- Displacement, velocity, acceleration sensor. They are used primarily for status and physical collection of CNC machine tools. Such as Fanuc system, we based on the FOCAS2 interface to write programs in order to achieve the related data real-time acquisition.

- Power sensor. This type of signal is mainly used for CNC equipment energy consumption management, through coordinated by matching with the manufacturers to achieve such signals in real time access.

- Acoustic emission sensors. They are mainly used for tool wear monitoring and management, these sensors require separately purchased and installed.

- To avoid system instability caused by different kinds of sensors, we design a plug-in architecture based on "abstract factory pattern"[20]. As shown in Figure 2.

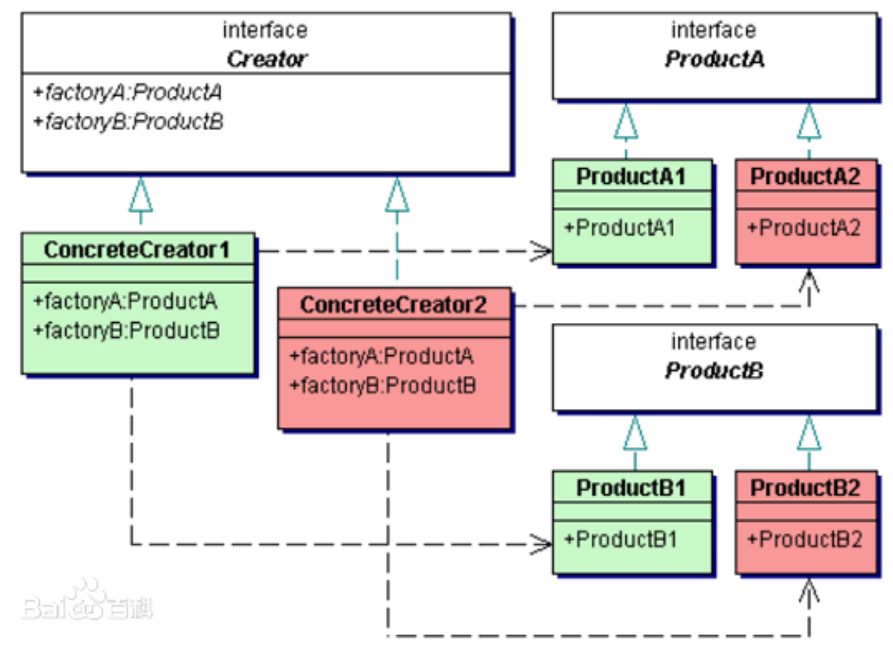

Figure 2. Abstract Factory Design Pattern

3.1.2. Real Time Control System Module: This module is responsible for real-time control of CNC equipment, such as abnormal shutdown, upload/download CNC programs. Programmatically varies with different devices, here also use the factory pattern design. 
Here is an example of Fanuc system via the FOCAS2 interface to transfer program, as shown in Figure 3.

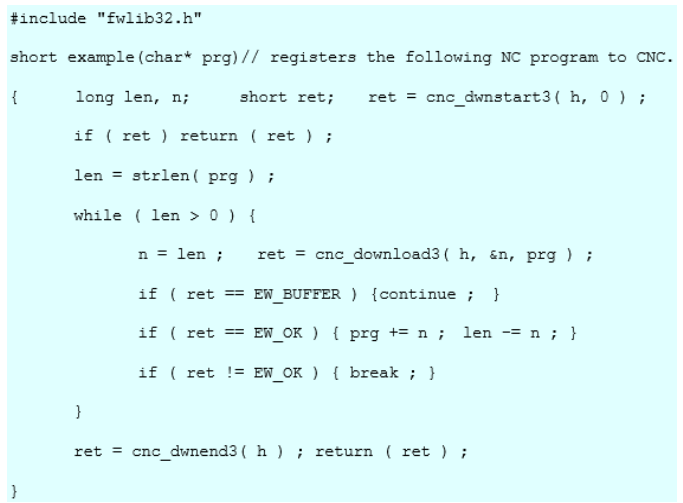

Figure 3. An Example of Fanuc System via the FOCAS2 Interface to Transfer Program

3.1.3. Data Acquisition Services Module: We develop data collection service factory class for different devices, to convert different bus technology for industrial Ethernet, different boundaries are converted to standard semantic model.
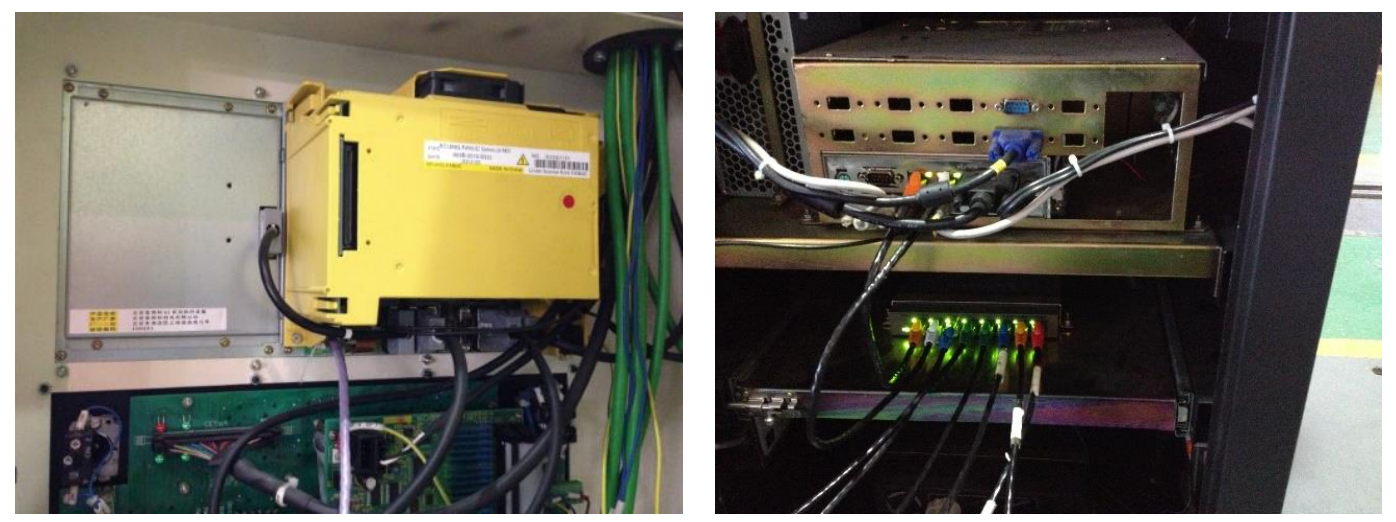

Figure 4. The Data Acquisition Services Module

\subsection{Gathering and Storage Layer}

This layer is mainly responsible for network transmission of data, data cleansing and data storage capabilities for upper management provides real-time, accurate and reliable data to support. This level contains the remote data acquisition module, service module, data cleansing and data storage module.

3.2.1. Remote Data Acquisition Module: This module has the following two functions:

- Providing metadata management in order to achieve registration of data acquisition services module. The registration information includes the IP address of the network, computer numerical control system model, the data service class name, frequency, and so on.

- Establishing a system daemon in order to automatically read at the set frequency.

3.2.2. Data Cleansing Service Module: This module receives data from the remote data acquisition module, then check the data reliability. Industrial environments can result in Ethernet transmission signal noise, thereby affecting the upper module to determine accuracy, so we carry out noise reduction. 
To the manipulator displacement signal acquisition, for example, if the collection cycle is less than $10 \mathrm{~ms}$, the multiple manipulator displacement data appears incidental phenomenon of chaos. It results that a robotic arm moves abnormalities in a simulation module. To avoid this behavior, set the data difference threshold, detect noise and automatically filter it

\subsection{Management and Control Layer}

3.3.1. System Configuration Module: This module provides complete management capabilities for total system, including the following aspects.

- Providing metadata management in order to achieve registration of data acquisition services module. The registration information includes the IP address of the network, computer numerical control system model, the data service class name, frequency, and so on.

- It through the product collection to retrieve the appropriate configuration information, including process cards, NC programs, parts, drawings, and so forth, and transmits the information to the current configuration directory, and notice the underlying module upload these files to NC Line.

- It provides role-based permissions for configuration management capabilities.

- It provides disaster recovery mechanisms such as database backup and recovery management.

- It provides version online update capabilities, $3 \mathrm{G}$ or cable networks for Web-based remote updates.

3.3.2. Online Diagnosis Module: This module builds the equipment fault diagnosis model and tool breakage monitoring model to reduce production downtime.

- It parses the equipment State-related time series information, such as the run rate, feed speed, spindle speed, temperature of machine tools, and so on, according to the existing thresholds, recognizes the State of health and active alerts.

- It will analyze time series information, associated with power and acoustic emission signals, determine the time-domain, frequency domain trends and thresholds that are already identifying tool breakage and proactive alerts.

3.3.3. Process Monitoring Module: This module can import external production orders, implement complete quality inspection, export the report data and simulation of machining process.

- It can import external systems production order based on the XML format, and can also be manually entered into the production order.

- It can be combined with processing quantity and testing equipment collected test results, statistics of the number of completion and number of waste. Because of the complexity of discrete manufacturing, it provides an interface to enter information related to quality.

- It can export line status information, including implementation of the production orders, equipment operation, tool breakage, QC data and other related information based on XML or Excel formats.

- It can process simulation based on computer animation techniques, in order to reflect the processing status in real time. 


\section{Application Cases}

Through cooperation with a leading machine tool manufacturer in China, HNCS prototype system has been selected for group control system pre-installed and has sold 19 NC line until now.

\subsection{General Information about the Case}

This paper has developed the HNCS prototype system, which is been applied in a large State-owned enterprise in Wuxi, China. The customer purchased 2 NC lines, includes automatic Hopper, truss robots and CNC machining equipment (Figure 5).

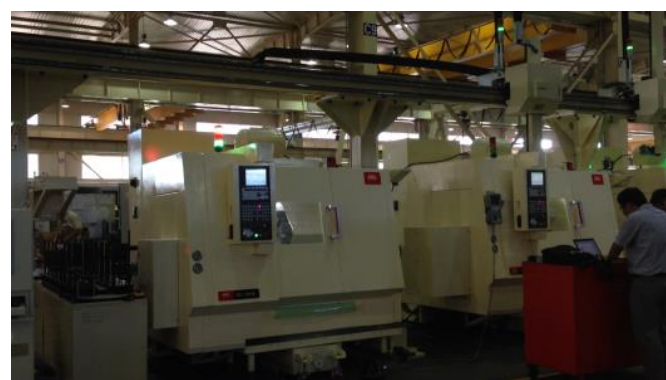

Figure 5. HNCS System Network Topology

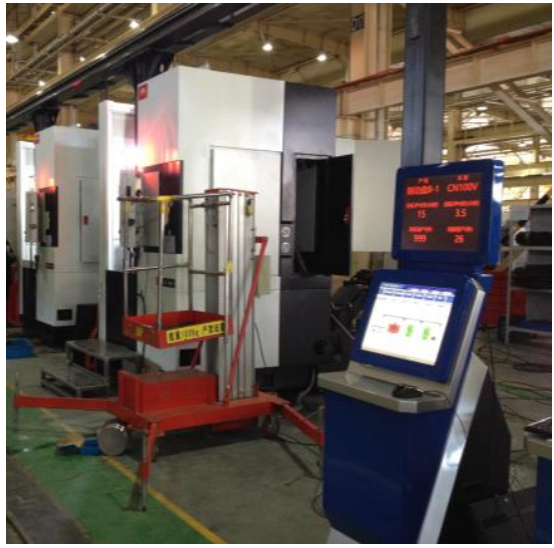

Figure 6. Real Photos of HNCS Terminal Equipment

Figure 6 is a live photo, and Figure 7 is the primary interface system.
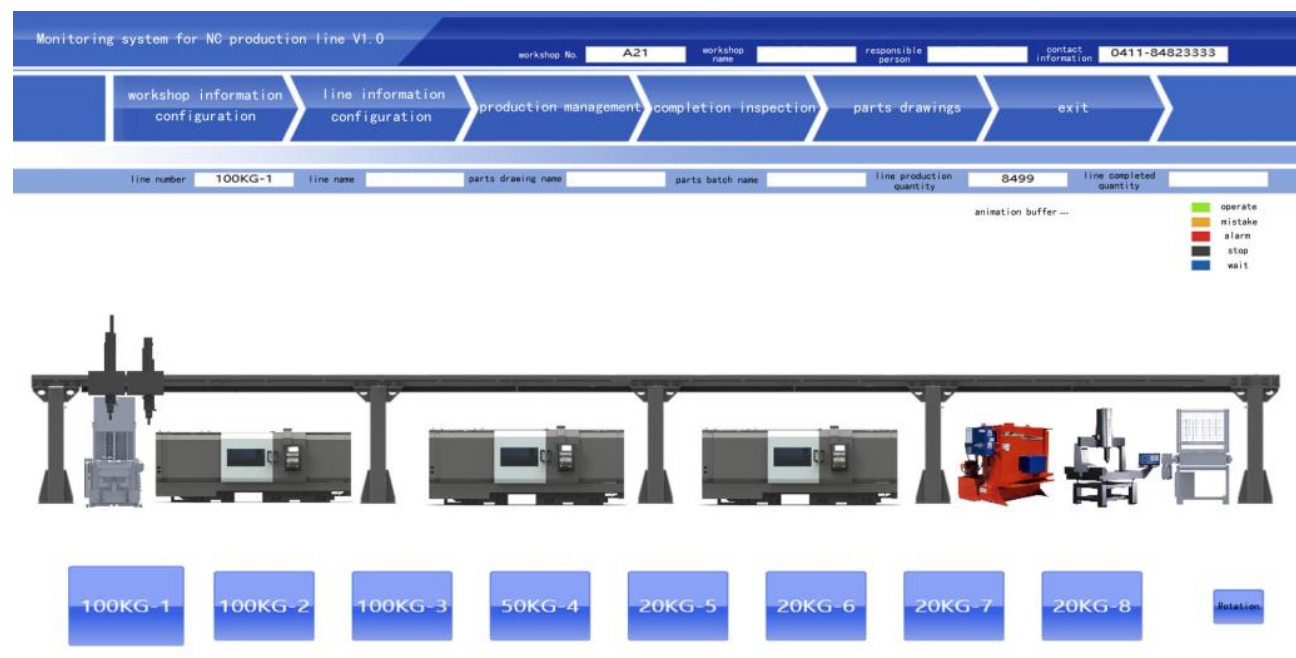

Development organization: Dalian University Technology \& Shenyang machine tool Refco Group Lte

Figure 7. HNCS the System's Primary Interface

\subsection{Monitoring and Simulation Section}

It calculates the actual force of the cutter (Figure 8), based on the collected power and acoustic emission signal, and capable of predicting tool life (Figure 9). 


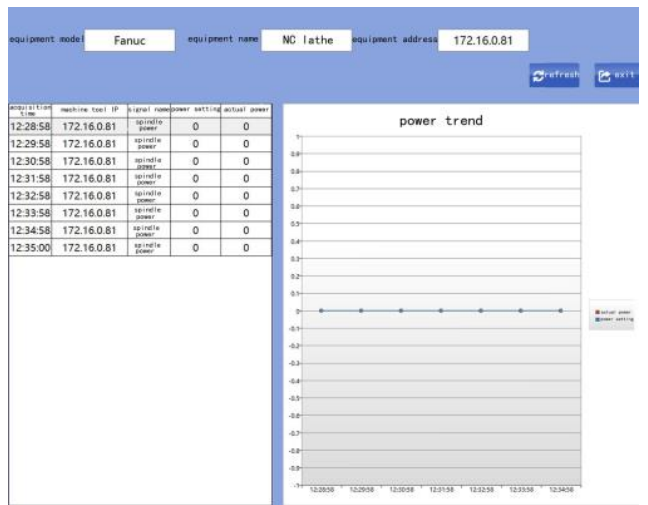

Figure 8. The Actual Force of the Cutter

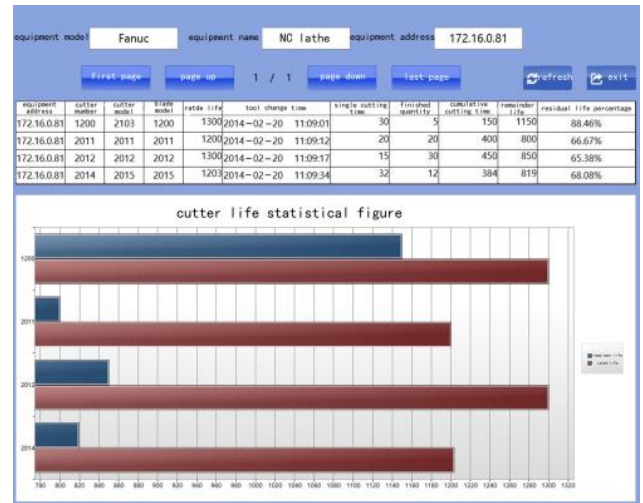

Figure 9. Tool Life Prediction

\section{Conclusions}

Discrete manufacturing system as a highly complex nonlinear dynamic system, modeling and management issues have been the focus of academic research and problems. Using the sensor network, we connect NC production line components, CNC production lines based on information technology upgrades for the CPS sense of intelligent robot system. These efforts offer a new solution to solve real-time, intelligent control for discrete manufacturing system.

We designed and developed a prototype system and applied in Chinese enterprises, a preliminary examination of this paper feasibility and practicability.

\section{Acknowledgements}

This work was financially supported by the Fundamental Research Funds for the Central Universities (DUT14QY27), National Science and Technology Major Project of the Ministry of Science and Technology of China (2013ZX04012071) and FAW Jiefang Automotive Co. Ltd. of Wuxi.

\section{References}

\subsection{Journal Articles}

[1] R. Ning, J. Liu and C. Tang, "Modeling and simulation technology in digital manufacturing [J]", Chinese Journal of mechanical engineering, vol. 7, (2006), pp. 132-137.[In Chinese]

[2] X. Li, "Modeling and simulation of production line based on the Flexsim [J]", Mechanical engineer, vol. 6, (2007), pp. 90-91. [In Chinese]

[3] Y. Yang, C. Lu and Z. Li, "Assembly processes based on Petri nets modeling and simulation analysis [J]", Mechanical engineer, vol. 11, (2008), pp. 88-90. [In Chinese]

[4] Y. Chao and H. Liu, "BIW welding robot path optimization and simulation [J]", China mechanical engineering, vol. 4, (2010), pp. 442-445. [In Chinese]

[5] F. Wanga and H. Xiang, "Flexsim software production line simulation based optimization [J]", Mechanical engineer, vol. 11, (2010), pp. 88-89. [In Chinese]

[6] C. Zhang, X. Fana nd T. Li, "Optimization configuration the number of various types of AGV based on simulation optimization method [J]", China mechanical engineering, vol. 14, (2011), pp. 680-1685. [In Chinese]

[7] S. Wang, B. Zhang, F. Liu and J. Huang, "Assembly line online quality control system based on intelligent agents [J]”, Chinese Journal of mechanical engineering, vol. 6, (2003), pp. 145-150. [In Chinese]

[8] Z. Liu, Z. Jiang, G. Maogen, H. Jin and M. Zhang, "Field data acquisition and process monitoring for Assembly [J]", Mechanical engineer, vol. 4, (2009), pp. 52-54. [In Chinese]

[9] L. Xi, "Detecting casing production line based on PLC control system design of China [J]", Mechanical engineering and automation, vol. 2, (2010), pp. 162-163+165. [In Chinese] 
[10] G. Yin, C. Zhang and R. Liu, "Distributed computer control system for sand casting production line [J]", China mechanical engineering, vol. 1, (1995), pp. 57-60+78. [In Chinese]

[11] Z. Zhang and T. Li, "Coal dense medium system and design of PLC monitoring and research [J]", Mechanical engineering and automation, vol. 3, (2008), pp. 151-153. [In Chinese]

[12] F. Wang, "Mechanical and electrical control system based on PLC and fieldbus [J]", Mechanical engineering and automation, vol. 2, (2013), pp. 208-209. [In Chinese]

[13] Z. Wang, D. Yu, X. Zhang, Y. Hu, P. Li and M. Chen, "Research of CNC system for reliable fieldbus communication mechanisms [J]", Chinese Journal of mechanical engineering, vol. 3, (2011), pp. 152-158+165. [In Chinese]

[14] Z. Wang and G. Zhu, "New research on open numerical control system based on fieldbus [J]", China mechanical engineering, vol. 4, (2001), pp. 36-38+5. [In Chinese]

[15] Z. Wang and L. Xie, "Summary of the research on the physics of information fusion system [J]", Journal of automation, vol. 10, (2011), pp. 1157-1166. [In Chinese]

[16] E. Lee, "Computing Foundations and Practice for Cyber-Physical Systems: a Preliminary Report", Technical Report UCB/EECS-2007-72, University of California, USA, (2007).

[17] R. Baheti and H. Gill, "Cyber-physical systems. The Impact of Control Technology", Washington D. C., USA: IEEE, (2011), pp. 161-166.

[18] CPS Steering Group, Cyber-physical systems execu-tive summary [Online], available: http://precise.seas.upenn.edu/events/iccps11/ doc/CPS-Executive-Summary.pdf, June 4, 2011.

[19] J. Lin, S. Sedigh and A. Miller, "A general framework for quanti-tative modeling of dependability in cyber-physical systems: a proposal for doctoral research", In: Proceedings of the 33rdAnnual IEEE International Computer Software and Appli-cations Conference. Seattle, USA: IEEE, (2009), pp. 668, 671.

[20] J. Vlissides, R. Helm and R. Johnson, "Design patterns: Elements of reusable object-oriented software", Reading: Addison-Wesley, vol. 49, (1995), p. 120.

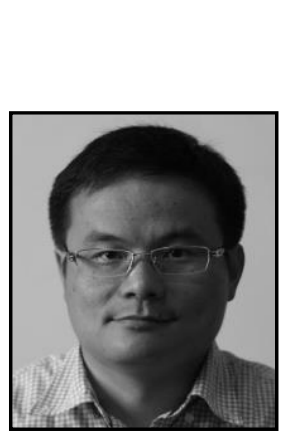

\section{Author}

Feng Qian, he was born in Huainan, Anhui province, in April 2, 1977. He devoted to the research of mechanical design and theory.He graduated from the Dalian University of Technology where is located in Dalian, China and obtained a master's degree in the university in 2003. Since 2003, he has worked as a lecturer in the Dalian University of Technology. 